\title{
Real-time motion planning in the presence of moving obstacles
}

\author{
Tim Mercy, Wannes Van Loock and Goele Pipeleers \\ KU Leuven, BE-3001 Leuven, Belgium \\ Department of Mechanical Engineering, Division PMA \\ Tim.Mercy@kuleuven.be
}

\section{Introduction}

Reasons to automate processes vary widely, from relieving humans from repetitive tasks such as harvesting fields over improving accuracy in robotic surgery to increasing the speed of product assembly. For these purposes industry uses autonomous motion systems like robotic arms, autonomous guided vehicles and drones. Safe operation of any autonomous system demands a collision-free motion trajectory at every time instant. Since the environment is generally variable, real-time trajectory generation is required.

This abstract presents a method to calculate time-optimal motion trajectories for autonomous systems moving through an environment with both stationary and moving obstacles, by using numerical optimization. To transform this motion planning problem into a small dimensional optimization problem, suitable for a real-time implementation, the approach (i) uses a spline parameterization of the motion trajectory; and (ii) exploits spline properties to reduce the number of constraints [1]. By solving the resulting optimization problem with a receding horizon it is possible to deal with variations in the environment.

The method is experimentally validated on a KUKA youBot. The average solving time of the optimization problem in the experiments $(0.05 \mathrm{~s})$ is sufficiently fast for correcting deviations from the initial trajectory.

\section{Methodology}

This abstract builds on [1], which uses a B-spline parameterization for the motion trajectory. This work only consideres static environments containing circular obstacles. The proposed method extends the approach with an efficient solution to include anti-collision constraints for convex obstacles, which is based on the separating hyperplane theorem [2]. Since both the system and the obstacles can move, the separating hyperplanes are allowed to be time-varying, by parameterizing them as a B-spline. Furthermore, the method takes into account the latest information about the (uncertain) environment by solving the problem with a receding horizon. The real-time motion planning provides implicit position feedback and only additional wheel velocity feedback is required to steer the system. Finally, the method accounts for obstacle movements by using a linear prediction of the obstacle position, which is based on the obstacle's measured velocity.
The resulting optimization problem contains initial and final conditions on the system's state, kinematic constraints, and anti-collision constraints, which are described by separating hyperplanes. The goal is to find the minimal motion time and determine the corresponding spline coefficients of the motion trajectory.

The developed method generates a time-optimal point-topoint trajectory which steers an autonomous motion system along circular and rectangular, stationary and moving obstacles. The method is included into a motion planning toolbox, which allows users to easily implement, solve and simulate motion planning problems for both single agent and multi-agent systems.

\section{Results}

Figure 1 shows a test result for a youBot which has to pass two stationary rectangular obstacles. Every cross denotes a new iteration of the method, every curve denotes a calculated trajectory. The average calculation time per iteration was $0.05 s$, the maximum time was $0.45 s$. This figure proves that feedforward velocity setpoints suffice for trajectory following and that no further control methods are necessary.

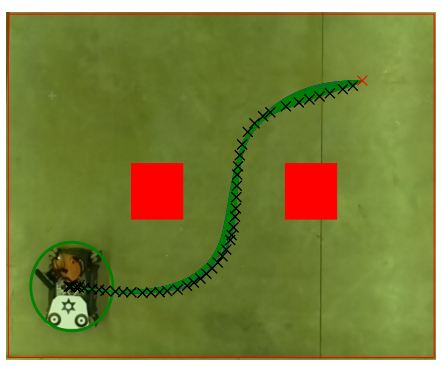

Figure 1: Time-optimal motion of the youBot

Acknowledgement IWT ICON project Sitcontrol: Control with Situational Information, IWT SBO project MBSE4Mechatronics: Model-based Systems Engineering for Mechatronics, FWO project G0C4515N: Optimal control of mechatronic systems: a differential flatness based approach. This work also benefits from K.U.LeuvenBOF PFV/10/002 Center-of-Excellence Optimization in Engineering (OPTEC), from the Belgian Programme on Interuniversity Attraction Poles, initiated by the Belgian Federal Science Policy Office, and from K.U.Leuven's Concerted Research Action GOA/10/11 "Global real-time optimal control of autonomous robots and mechatronic systems".

[1] W. Van Loock, G. Pipeleers, and J. Swevers, "B-spline parameterized optimal motion trajectories for robotic systems with guaranteed constraint satisfaction," Mechanical Sciences, vol. 6, no. 2, pp. 163-171, 2015.

[2] S. Boyd and L. Vandenberghe, Convex Optimization. Cambridge, UK: Cambridge University Press, 2004 